\title{
High Accuracy Microwave Frequency Measurement Based on Single-Drive Dual- Parallel Mach-Zehnder Modulator
}

Zhao, Ying; Deng, Lei; Pang, Xiaodan; Yu, Xianbin; Zheng, Xiaoping; Zhou, Bingkun; Tafur Monroy, Idelfonso

\section{Published in:}

Proceedings of the European Conference on Optical Communication (ECOC) 2011

Publication date:

2011

Document Version

Publisher's PDF, also known as Version of record

Link back to DTU Orbit

Citation (APA):

Zhao, Y., Deng, L., Pang, X., Yu, X., Zheng, X., Zhou, B., \& Tafur Monroy, I. (2011). High Accuracy Microwave Frequency Measurement Based on Single-Drive Dual-Parallel Mach-Zehnder Modulator. In Proceedings of the European Conference on Optical Communication (ECOC) 2011 (pp. We.10.P1.118). Optical Society of America. http://www.ecoc2011.org/

\section{General rights}

Copyright and moral rights for the publications made accessible in the public portal are retained by the authors and/or other copyright owners and it is a condition of accessing publications that users recognise and abide by the legal requirements associated with these rights.

- Users may download and print one copy of any publication from the public portal for the purpose of private study or research.

- You may not further distribute the material or use it for any profit-making activity or commercial gain

- You may freely distribute the URL identifying the publication in the public portal 


\title{
High Accuracy Microwave Frequency Measurement Based on Single-Drive Dual-Parallel Mach-Zehnder Modulator
}

\author{
Ying Zhao ${ }^{1,2 \dagger}$, Lei Deng ${ }^{2,3}$, Xiaodan Pang ${ }^{2}$, Xianbin Yu ${ }^{2}$, Xiaoping Zheng ${ }^{1 \ddagger}$, \\ Bingkun Zhou ${ }^{1}$ and I. Tafur Monroy ${ }^{2}$ \\ ${ }^{1}$ Department of Electronic Engineering, Tsinghua National Laboratory for Information Science and Technology, \\ Tsinghua University, 100084 Beijing, China. \\ ${ }^{2}$ DTU Fotonik, Technical University of Denmark, DK-2800, Kgs. Lyngby, Denmark. \\ ${ }^{3}$ School of Optoelectronics Science \& Engineering, HuaZhong Univerity of Science \& Technology., Wuhan, China. \\ †yinzh@fotonik.dtu.dk $\ddagger x p z h e n g @ m a i l . t s i n g h u a . e d u . c n$
}

\begin{abstract}
A novel approach for broadband microwave frequency measurement based on bias manipulation of a dual-parallel Mach-Zehnder modulator is proposed and experimentally demonstrated. A $10^{-3}$ relative error verifies a significant accuracy improvement by this method.
\end{abstract}

OCIS codes: (060.5625) Radio frequency photonics, (350.4010) Microwaves.

\section{Introduction}

Microwave photonics has driven and facilitated various kinds of applications for its superiority on potential highspeed and parallel microwave signal processing capability [1]. For electronic warfare and defense applications, carrier frequency measurement in optical manners can be a promising solution for intercepted signal processing where a high accuracy and large measurement range is required. The primary advantages of photonics-assisted microwave frequency measurement are the broadband and real-time operation of lightwave signals. Generally, the frequency measurement can be realized by comparing the power of two microwave signals experiencing different dispersion-induced fading effects in the optical link. Recently, the frequency-to-power mapping technique has been proposed and investigated by different methods [2-4]. Complementary filtering implemented by microwave photonic filters [2] are generally adopted while other schemes using multiple laser sources or modulators are developed [3,4]. The major limitation of previous methods is the comparatively large relative measurement error (several percent), which prevents them from being used in high accuracy applications.

In this paper, we propose and demonstrate a novel approach for high accuracy frequency measurement using a single-drive dual parallel Mach-Zehnder modulator (SD-DPMZM). By tuning one of the bias points of the SDDPMZM, the power fading characteristics of the unknown microwave signal changes, which thereby results in the change of measurement accuracy. Therefore, the highest accuracy point can be obtained by flexible manipulating the modulator bias point. The experiment verifies the improved accuracy of this approach by performing a $\sim 10^{-3}$ relative error within a $10 \mathrm{GHz}$ measurement range.

(a) SD-DPMZM

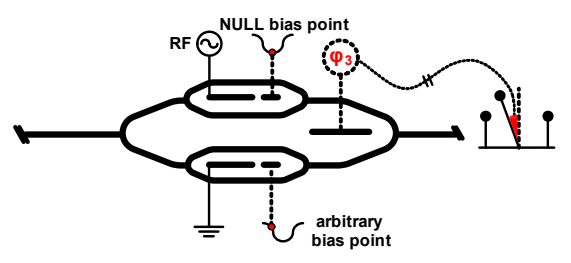

(b)

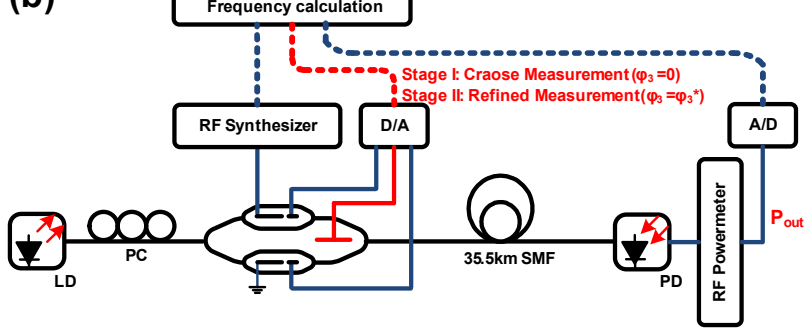

Fig. 1. (a) Bias arrangement of a SD-MZM for frequency measurement. (b) Experimental setup of a high accuracy frequency measurement system. LD: laser diode. PC: polarization controller. PD: photodiode. A/D and D/A: analog to digital converter and digital to analog converter. 


\section{Principle}

Fig.1(a) shows the bias arrangement of a DPMZM for frequency measurement purpose. The structure of the DPMZM contains two sub-MZMs, MZM1 and MZM2, lying in parallel on two arms of MZM3, which controlled only by its bias voltage without drive. In our case, MZM1 is biased at the minimal transmission point (NULL point) and driven by the unknown RF signal to perform carrier suppressed double sideband (CSDSB) modulation. MZM2 is remained un-modulated and arbitrarily biased to let the optical carrier propagate through. This asymmetrical arrangement is named single-drive of a DPMZM or CSDSB+Carrier modulation scheme [5]. The phase difference $\varphi_{3}$ between the optical carrier and two first-order sidebands is determined by the bias point of the MZM3, which also gives a RF $\varphi_{3}$-dependent power fading after dispersive transmission. As shown in Fig.1(b), the output RF power can be given by

$$
P_{\text {out }}\left(\varphi_{3}\right) \propto \cos ^{2}\left(\frac{\beta_{2} \cdot L}{2} \Omega^{2}+\varphi_{3}\right)
$$

where $\beta_{2}$ and $L$ are the dispersion coefficient and the length of the dispersive link, respectively. $\Omega$ is the angular frequency of the unknown RF signal. To eliminate undesirable effects caused by the non-flat frequency response of components in the link, a relative power comparison function (PCF) is introduced to construct RF frequency-to-power mapping, which can be expressed as $\operatorname{PCF}\left(\varphi_{3}\right)=P_{\text {out }}\left(\left.\varphi_{3}\right|_{t_{1}}\right) / P_{\text {out }}\left(\left.\varphi_{3}\right|_{t_{2}}=\left.\varphi_{3}\right|_{t_{1}}+\frac{\pi}{2}\right)$, where $\left.\varphi_{3}\right|_{t_{1}}$ and $\left.\varphi_{3}\right|_{t_{2}}$ are bias phases of twice measurements at time $t_{1}$ and $t_{2}$ and the phase difference between these measurements is fixed at $\pi / 2$. Therefore the unknown frequency is determined by

$$
\Omega\left(\left.\varphi_{3}\right|_{t_{1}}\right)=\sqrt{\frac{2\left(\operatorname{arccot}(\sqrt{P C F})-\left.\varphi_{3}\right|_{t_{1}}\right)}{\beta_{2} \cdot L}}
$$

Since $\varphi_{3}$ is an inherent parameter of the DPMZM and only dependent on the bias of the MZM3, we can flexibly choose $\left.\varphi_{3}\right|_{t_{1}}$ to achieve a potential high accuracy point implied by Eq.(2). If we choose the bias point

$$
\left.\varphi_{3}^{*}\right|_{t_{1}}=-\frac{\beta_{2} \cdot L}{2} \Omega^{2}
$$

the derivative $\left.\frac{\partial \Omega}{\partial P C F}\right|_{\varphi_{3}^{*}}=0$, which means the measurement frequency is non-sensitive to PCF error at all. The highest accuracy can be achieved by this deliberate bias manipulation.

\section{Experimental setup}

Fig.1(b) shows the high accuracy frequency measurement system using the proposed approach. The unknown RF signal is modulated onto an optical carrier at $1549.8 \mathrm{~nm}$ based on a SD-DPMZM. The optical signal is launched into a $35.5 \mathrm{~km}$ single mode fiber (SMF) and then detected by a photodiode. The power of the dispersion-induced fading signal is monitored by a RF powermeter. The system is running automatically by employing program-based GPIB instrument control interface implemented in MATLAB. The frequency measurement procedure is divided into two stages, I) the coarse measurement and II) the accuracy refining measurement. In the stage I, the coarse frequency $\tilde{\Omega}$ is estimated based on Eq.(2) with the fixed bias phase $\varphi_{3}=0$, which means the high accuracy requirement (Eq.(3)) is not satisfied in this case. The main purpose of the coarse measurement stage is to lead the bias point to approach the requirement of Eq.(3) in the stage II. By using the coarse measured frequency $\tilde{\Omega}$, we can approximately calculate the bias point $\varphi_{3}^{*}$ for highest accuracy using Eq.(3). The measurement system then switches the DC bias of the MZM3 to $\varphi_{3}^{*}$ and refines the measurement process. In our experiment, the stage II is executed iteratively for ten times and the output values are averaged as the final frequency estimation.

\section{Experimental results}

Fig.2 shows the coarse measurement results with $\varphi_{3}$ fixed at 0 . The PCF value with respect to the reference frequencies for different RF input powers is shown in Fig.2(a). The first power fading notch is at $\sim 10 \mathrm{GHz}$, which implies the unambiguous frequency measurement range is $\sim 10 \mathrm{GHz}$. This range can be adjusted by changing the amount of the optical link dispersion. Fig.2(b) shows the measured frequencies obtained from Eq.(2) as well as reference frequencies for different input power levels. The errors between the measurement and the reference are shown in Fig.2(c). The error curve drops significantly when the reference frequency is close to $10 \mathrm{GHz}$ because for a $35.5 \mathrm{~km} \mathrm{SMF}$, the highest accuracy requirement of the bias point is $\varphi_{3}^{*} \doteq 0$, almost got satisfied in the coarse measurement case. Fig. 3 shows the high accuracy measurement results based on the coarse pre-measurement for a 10dBm RF input. Fig.3(a) shows the error limits of the ten times iterative refined measurements. The absolute error fluctuation is less than $5 \mathrm{MHz}$, which shows the stability of the refined measurement process. The absolute error comparison between the coarse and refined measurements is shown in Fig.3(b). By performing high accuracy measurement, the average uncertainty 
of the measured frequency can be tremendously decreased from $118 \mathrm{MHz}$ to $5.4 \mathrm{MHz}$. Fig.3(c) shows the refined measurement error with the MZM3 bias voltage drift for $10 \mathrm{GHz}$ reference frequency considering the non-ideal biasing characteristics of the DPMZM. In this case, the bias drift is emulated by intentionally inducing a deviation from $\varphi_{3}^{*}$. A $\sim 60 \mathrm{MHz} / \mathrm{V}$ degradation of measurement performance is observed.

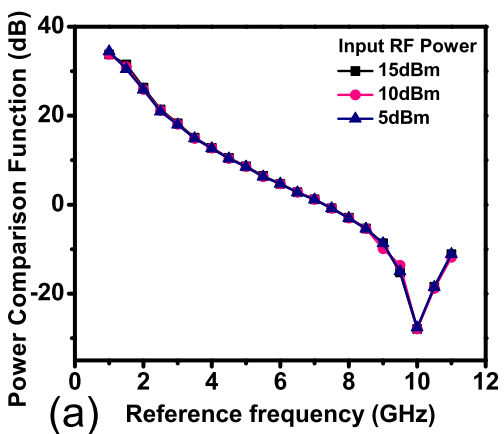

(a) Reference frequency $(\mathrm{GHz})$

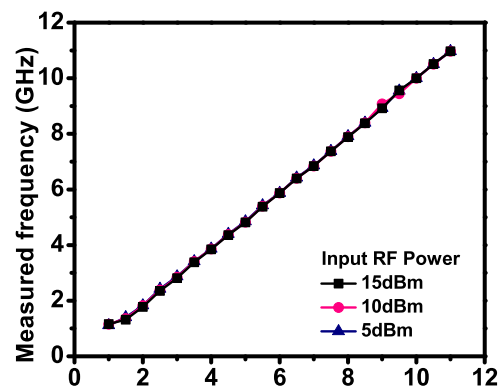

(b) Reference frequency (GHz)

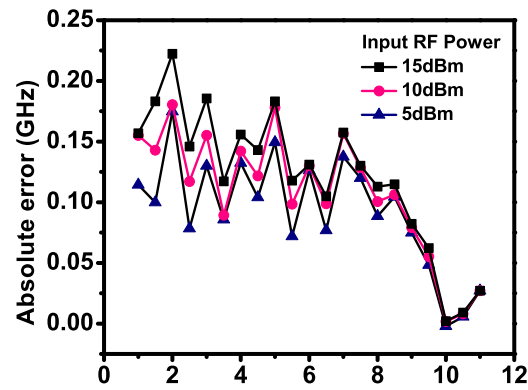

(C) Reference frequency $(\mathrm{GHz})$

Fig. 2. Frequency measurement results of coarse stage. (a) PCF value versus input RF frequency for different RF power. (b) Measured RF frequency versus input reference frequency for different RF power. (c) Absolute measurement error versus input reference frequency for different RF power.

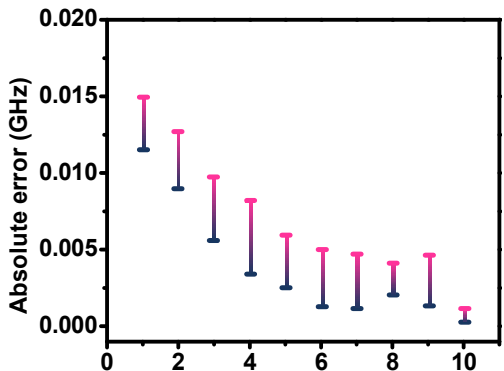

(a) Reference frequency $(\mathrm{GHz})$

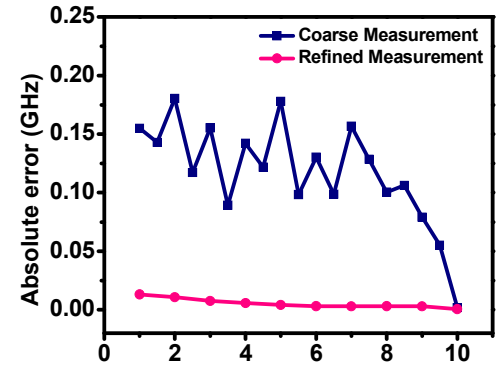

(b) Reference frequency (GHz)

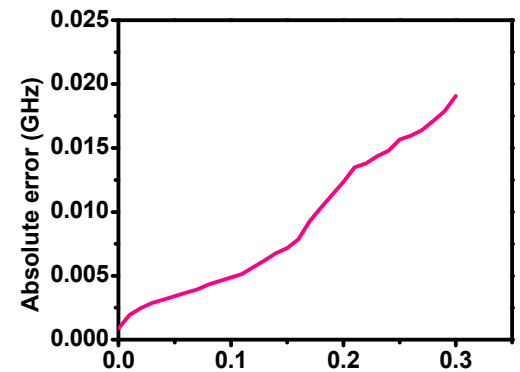

(c) Bias drift voltage (V)

Fig. 3. Frequency measurement results of refined stage. (a) Error range for ten times refined measurements. (b) Error comparison of coarse and refined measurement. (c) Absolute error effected by the MZM3 bias drift.

\section{Conclusions}

A novel scheme for high accuracy broadband microwave frequency measurement is proposed, theoretically analyzed and experimentally demonstrated. By using a SD-DPMZM and performing a two-stage measurement, this method shows an improved relative error of $10^{-3}$.

\section{Acknowledgements}

This work was supported in part by National Nature Science Foundation of China (NSFC) under grant No. 60736003, 61025004, 61032005 and National 863 Program under grant No. 2009AA01Z222, 2009AA01Z223.

\section{References}

1. J. Yao, “Microwave Photonics," IEEE J. Lightw. Technol., 27, 314-335 (2009).

2. S. Fu, J. Zhou, P. P. Shum and K. Lee, "Instantaneous microwave frequency measurement using programmable differential group delay (DGD) modules," IEEE Photonics J., 2, 966-973 (2010).

3. H. Chi, X. Zou and J. Yao, "An approach to the measurement of microwave frequency based on optical power monitoring," IEEE Photon. Technol. Lett., 20, 1249-1251 (2008).

4. X. Zou, S. Pan and J. Yao, "Instantaneous microwave frequency measurement with improved measurement range and resolution based on simultaneous phase modulation and intensity modulation," IEEE J. Lightw. Technol., 27, 5314-5318 (2009).

5. S. Li, X. Zheng, H. Zhang and B. Zhou, "Dispersion induced fading frequency shifting technology in Radioover-Fiber link," in Proceedings of 2010 IEEE Topical Meeting on Microwave Photonics (MWP), 321-322. 